This item was submitted to Loughborough's Research Repository by the author.

Items in Figshare are protected by copyright, with all rights reserved, unless otherwise indicated.

\title{
A methodology for modeling HVAC components using evolving fuzzy rules
}

PLEASE CITE THE PUBLISHED VERSION

http://dx.doi.org/10.1109/IECON.2000.973158

PUBLISHER

(c) IEEE

VERSION

AM (Accepted Manuscript)

LICENCE

CC BY-NC-ND 4.0

REPOSITORY RECORD

Angelov, Plamen, Richard A. Buswell, Victor I. Hanby, and Jonathan A. Wright. 2019. "A Methodology for Modeling HVAC Components Using Evolving Fuzzy Rules”. figshare. https://hdl.handle.net/2134/10149. 
This item was submitted to Loughborough's Institutional Repository (https://dspace.lboro.ac.uk/) by the author and is made available under the following Creative Commons Licence conditions.

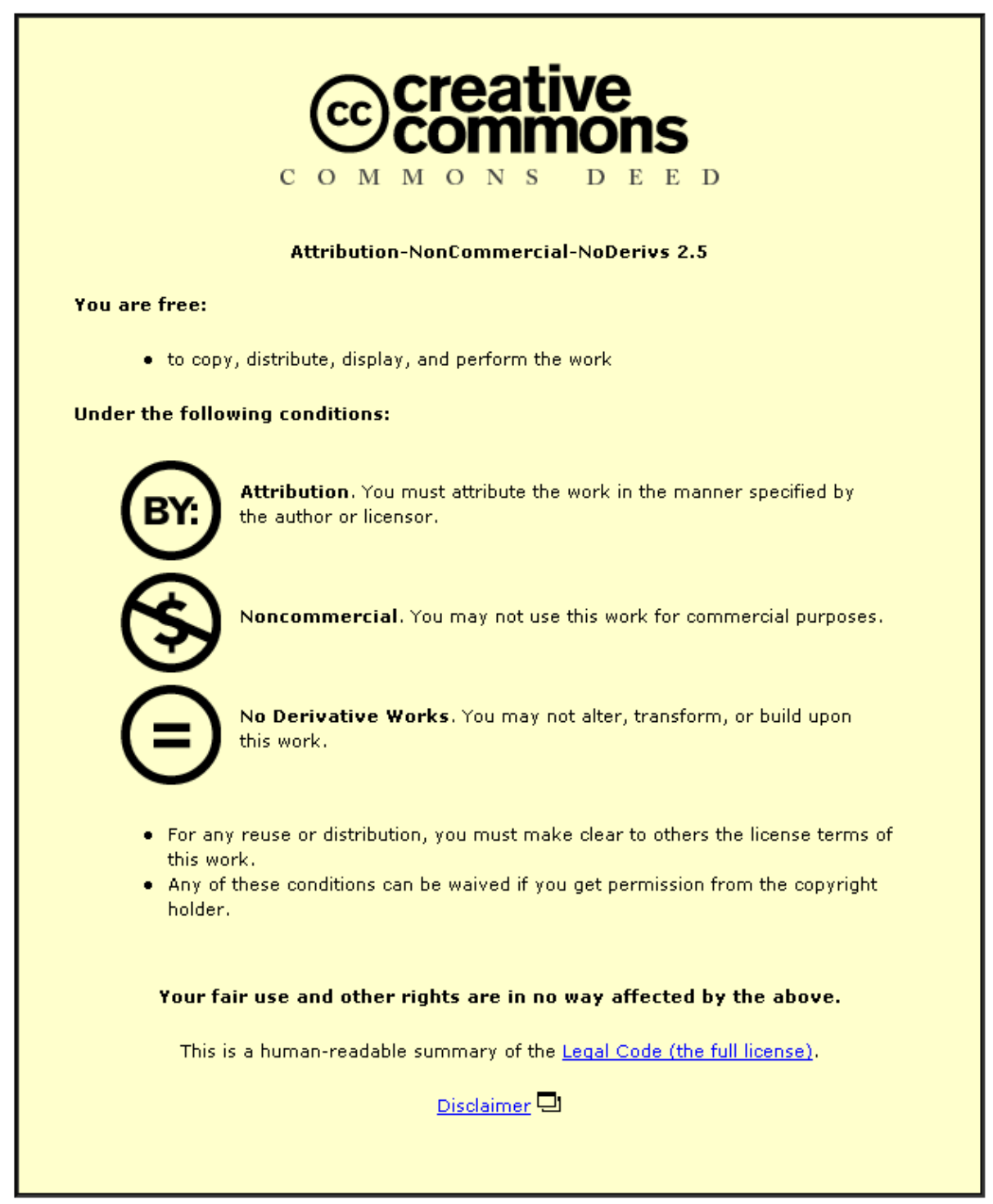

For the full text of this licence, please go to: http://creativecommons.org/licenses/by-nc-nd/2.5/ 


\title{
A Methodology for Modeling HVAC Components using
}

\author{
Evolving Fuzzy Rules
}

\author{
P. P. Angelov, V. I. Hanby, R. A. Buswell and J. A. Wright
}

\begin{abstract}
A methodology for the evolutionary construction of fuzzy rule-based (EFRB) models is proposed in the paper. The resulting models are transparent and existing expert knowledge could easily be incorporated into the model (both at initialisation stages and during its generation). An additional advantage of the model is represented by the economy in computational effort in generating the model output. A new encoding mechanism is used that allows the fuzzy model rule base structure and parameters to be estimated from training data without establishing the complete rule list. It uses rule indices and therefore significantly reduces the computational load. The rules are extracted from the data without using a priori information about the inherent model structure. It makes EFRB models as flexible as other types of 'black-box' models (neural networks, polynomial models etc.) and in the same time significantly more transparent, especially when only small subset of all possible rules is considered. This approach is applied to modelling of components of heating ventilating and air-conditioning (HVAC) systems. The EFRB models have potential applications in simulation, control and fault detection and diagnosis.
\end{abstract}

Keywords-Fuzzy Logic, Modelling, Genetic Algorithms, HVAC, Component Modelling.

\section{I.INTRODUCTION}

The computational demands of combined HVAC systems and building simulations can be considerable as these are often used for energy predictions over annual operational cycles. Fuzzy rule-based models as with other black-box approaches have the potential to reduce the computational demand of the simulation by mapping the inputs and outputs of the system components directly. In reality, processes associated with some typical components, such as boilers or compressors, can be too complex to be readily described by analytical methods and polynomial representations and recently neural networks [2] based on test data are employed. One disadvantage of black-box methods is the lack of transparency. Models using fuzzy rules can offer a high degree of transparency, but traditionally require the incorporation of a priori knowledge and subjective estimation to establish the rule base, i.e. the model structure. In many cases it is a complex and ambiguous process [6].

Recently genetic algorithms (GA) and neural networks have been used to extract an appropriate rule base from data [1], [3]-[5], [9], [12]-[13]. In [3] and [13] they are used for adjustment of parameters of membership functions only (parameter identification). In another group of papers the linguistic labels are assigned at the end of the identification process only [4]-[5], which makes these models quite close to neural networks, including the limited interpretability.

The limitation of these approaches has been that the exhaustive list of fuzzy rules is usually considered [9],[12]. The length of the chromosome there is determined on the basis of all possible combinations of linguistic variables, which hampers solving problems with realistic dimensions and makes models practically not interpretable.

An effective encoding approach [1] is used in this paper. It significantly minimises the length of the chromosome, which is also due to the use of realcoded GA. This permits simultaneous parameter and structure identification as well as the application of the approach to problems with realistic dimensions.

Unlike most of the black-box models and some of the fuzzy rule-based approaches, the knowledge extracted from the data is fully interpretable and could help for better understanding of the nature of the process being modelled. Expert knowledge could be added both at the initialisation step and during the identification process. Hybrid types of models composed of both fuzzy rules and crisp equations or inequalities could also be considered. The approach is applied to the modelling of axial fans as a components of an air conditioning system.

\section{EVOLVING FUZZY RULES}


Generally, fuzzy rule-based models consists of a number of rules of the following type, called Mamdani type [6]:

$$
\text { IF }\left(\mathrm{x}_{1} \text { is } \mathrm{X}_{1}\right) \text { AND...AND }\left(\mathrm{x}_{\mathrm{n}} \text { is } \mathrm{X}_{\mathrm{n}}\right) \mathbf{T H E N}(\mathrm{y} \text { is } \mathrm{Y})
$$

where $x_{i}$ is a fuzzy linguistic input variable;

$\mathrm{y}$ is the output variable;

$\mathrm{Y}$ is the fuzzy linguistic term of $\mathrm{y}$; $\mathrm{Y} \in\left\{\mathrm{Y}^{1}, \mathrm{Y}^{2}, \ldots, \mathrm{Y}^{\mathrm{m} 0}\right\}$;

$\mathrm{X}_{\mathrm{i}}$ is the fuzzy linguistic term or label of the $\mathrm{i}$-th input variable; $\mathrm{X}_{\mathrm{i}} \in\left\{\mathrm{X}_{i}^{1}, \mathrm{X}_{i}^{2}, \ldots, \mathrm{X}_{i}^{m i}\right\}$

A fuzzy set and its membership function define each linguistic label. Different types of membership functions are possible: Gaussian, triangular, trapezoidal, etc. [6].

For a specified number of linguistic variables and labels it is possible to determine the number of all possible fuzzy rules (R), which could be formulated out of them. Even if only combinations, in which each variable is participating are considered, this number could be extremely high for real problems, because of the so called curse of dimensionality [6],[9],[12]:

$\mathrm{R}=\prod_{\mathrm{j}=1}^{\mathrm{n}+1} m_{\mathrm{j}}$

where $m_{j}$ is the number of linguistic terms of the $j$-th linguistic variable.

It would be impossible to interpret such a model, even if it is generated automatically. Practically, significantly smaller number of rules (r) could be used, because of information redundancy [6]:

$\mathrm{r}<<\mathrm{R}$

Extraction of a set of rules has been made, generally, by the following two approaches: using neural networks or by GA. We explore the second one.

- GA could be considered as a driven stochastic search technique which imitate the process of natural selection. They are specifically appropriate for the problem we have to solve [7], because of their robustness, model structure independence, capacity to escape local minima.

The GA probes a set of trial points at every iteration. The trial set, called population, consists of several chromosomes, which comprises a number of genes. Each problem variable is coded into a gene. The modified version of the original binary GA [7], called real-coded GA or evolutionary algorithms
[10] makes problem definition more compact by representing each variable by a single gene:

TABLE I

REAL-VALUED CHROMOMOME

\begin{tabular}{|l|l|l|l|}
\hline$x_{1}^{i}$ & $x_{2}^{i}$ & $\cdots$ & ${ }_{\mathrm{n}}^{\mathrm{i}} \mathrm{x}$ \\
\hline
\end{tabular}

where $i=1,2, \ldots P ; P$ denotes the population size.

Part of the chromosomes from the current epoch is selected for reproduction. There is two operations, which are usually applied for producing new chromosomes: crossover and mutation. Mutation is a triggering from 0 to 1 and vice versa for the standard binary-coded GA [7]. Different schemes for mutation exist for real-coded GA [10].

\section{FUZZY RULES ENCODING}

Application of evolutionary technique for extraction of the fuzzy model requires an appropriate encoding of the fuzzy rules and their parameters. Encoding of all possible fuzzy rules into the chromosome as in [9],[12] is time consuming and can become an nonsolvable problem for problems with realistic dimension (>5 inputs and 7-9 linguistic labels). We propose to consider encoding of the indices of rules, which participate into the fuzzy model only. Their number is significantly smaller: normally some tens of rules are used and could be interpreted. Different encoding schemes could be used. The basic requirement is non-ambiguity (uniqueness) in coding and decoding. We introduce a simple encoding procedure, which assigns an index to every possible rule. A positive integer number represents each fuzzy rule. The genotype of the chromosome considered in our approach consists of two parts: indices of rules, which participate into the fuzzy model and their parameters:

TABLE II

GENOTYPE: LEFT PART REPRESENTS INDICIES OF RULES; Right ONE - MEMBERSHIP FunCTIONS' PARAMETERS \begin{tabular}{|l|l|l|l|l|l|l|l|}
\hline $\mathrm{I}_{1}$ & $\mathrm{I}_{2}$ & $\ldots$ & $\mathrm{I}_{\mathrm{K}}$ & $\mathrm{p}_{11}$ & $\mathrm{p}_{12}$ & $\ldots$ & $\mathrm{p}_{m_{i} n+1}$ \\
\hline
\end{tabular}

A two-stage coding scheme is adopted in this paper: first, we translate each linguistic label into a $\mathrm{L}$ based number (where $L=\max _{i=1}^{n+1}\left(m_{i}\right)$ is the maximal number of labels in all linguistic variables). 0 is assigned to the one marginal linguistic label, 1 to the next etc. As second stage, we transform the set of L-based numbers (codes of labels) into decimal integer positive number. They represent the index of the considered fuzzy rule:

$\mathrm{I}=\left(\mathrm{t}_{\mathrm{L}}^{1}, \mathrm{t}_{\mathrm{L}}^{2}, \ldots, \mathrm{t}_{\mathrm{L}}^{\mathrm{n}}\right)_{10}+1$ 
where I denotes the index of the fuzzy rule; $\mathrm{t}^{\mathrm{j}}, \mathrm{j}=1,2, \ldots, \mathrm{n}+1$ is code of the label $\mathrm{t}^{\mathrm{j}} \in\left[0 ; \mathrm{m}_{\mathrm{i}}-1\right]$

As an example the encoding of the following rule could be considered:

IF $\left(\mathrm{LV}_{1}\right.$ is High) AND ( $\mathrm{LV}_{2}$ is Very Low $)$ AND $\left(\mathrm{LV}_{3}\right.$ is Low $)$ THEN $\left(\mathrm{LV}_{4}\right.$ is Medium $)$

In this rule there are 4 linguistic variables (3 input and an output), $n=3$. Let the first input and the output have 3 linguistic terms (Low, Medium and $\mathrm{High}$ ) and all other variables have 5 linguistic terms (Very Low, Low, Medium, High and Very High). First, the codes of the used linguistic terms are determined:

$\mathrm{L}=\max (3,5,5,3)=5 ; \quad \mathrm{a}_{5}^{1}=2 ; \mathrm{a}_{5}^{2}=0 ; \mathrm{a}_{5}^{3}=1 ; \mathrm{a}_{5}^{4}=1$

Index of the fuzzy rule (5) is determined as a transformation to the integer with a decimal base according to (4):

$\mathrm{I}=\left(2{ }_{5} 0_{5} 1_{5} 1_{5}\right)_{10}+1=2 * 5^{3}+1 * 5^{1}+1 * 5^{0}+1=257$

Decoding process is an inverse of the coding one. First, the codes of linguistic labels are determined from the index of the rule as residuals in division by L:

$$
\begin{array}{ll}
{[(257-1) / 5]=51,} & \operatorname{Res}((257-1), 3)=1 ; \\
{[51 / 5]=10,} & \operatorname{Res}(51,3)=1 ; \\
{[10 / 5]=2 ;} & \operatorname{Res}(10,5)=0 ; \\
{[2 / 5]=0 ;} & \operatorname{Res}(2,5)=2 ;
\end{array}
$$

where Res(.) denotes residual in division of integers; [.] denotes integer result in division of integers.

Residual values determine in a unique way the fuzzy rule (5) from the index 257.

This effective encoding mechanism makes it possible to treat parameters of fuzzy membership functions as unknowns as well. Real-valued GA also minimises the chromosome representation and contributes to the compactness. Encoding of fuzzy rule parameters together with their structure into the same chromosome reveals a possibility for finetuning of the fuzzy rule-based models generated. It makes identification process more flexible and more independent on the subjectivity in structure determination. In the same time, certain degree of influence on the model structure is also possible. It could be realised by definition of parameters like the number of linguistic terms $(\mathrm{m})$, the maximal number of fuzzy rules considered (K), the pre-defined level of correlation ( $r$ ) and the type of the membership functions as well as using a priori knowledge in the initialisation. The number of fuzzy rules in the model could be finally smaller than $\mathrm{K}$ due to possible coincidence of some indices as well as due to appearance of zeros as rules indices (zero is left as an 'empty' index). Therefore $\mathrm{K}$ defines the upper bound of $\mathrm{k}$ - the number of used fuzzy rules. In the same time $\mathrm{K}$ could be significantly smaller than $\mathrm{R}$, which defines the number of all possible rules. Practically, some tens of rules are enough for reaching a pre-defined level of correlation and such number of rules is still interpretable. The number of linguistic labels considered $(\mathrm{m})$ is recommended to be $7 \pm 2(5,7$ or 9$)$ as closer to the human perception [6]. Values of correlation higher than 0.95 are seen as acceptable level of closeness between experimental and model outputs.

\section{EVOULTIONARY SEARCH PROCEDURE}

Numerical solution of both parameter and structural identification problems is sought by an evolutionary search procedure. The identification problem could be formulated as

To determine the fuzzy rules (represented by their indices) and their parameters such that to minimise the deviation between the model and the experimental outputs (represented by correlation):

$$
r \rightarrow \max
$$

subject to $(1)$

$$
1 \leq \mathrm{I}_{\mathrm{i}} \leq \mathrm{R} ; \quad \mathrm{i}=1,2, \ldots, \mathrm{K}
$$

$(j-1) \delta \leq p_{1 j} \leq \delta(j+1) ; 1=1,2, \ldots, n+1 ; j=1,2, \ldots m_{l}$

where $\delta=\frac{\overline{L V}-\underline{L V}}{m_{l}+1}$

$\underline{L V}_{l}$ is the lower bound of the 1-th linguistic variable;

$\overline{L V}_{l}$ is its upper bound .

It is important to note that fuzzy model (1) is considered as one of the constraints.

Evolutionary algorithm, which is applied for numerical solution of this problem, matches better the specifics of the considered problem:

- Vector of unknowns consists of integer (indices of fuzzy rules) and real (parameters of the membership functions) values, not binary ones;

- Real-valued GA supposes shorter chromosome, which allows simultaneous parameter and structure identification as well as solving problems with realistic dimensions.

The basic algorithm applied to our problem could be represented by the following pseudo-code.

\section{Begin}

Epoch $=0$; 


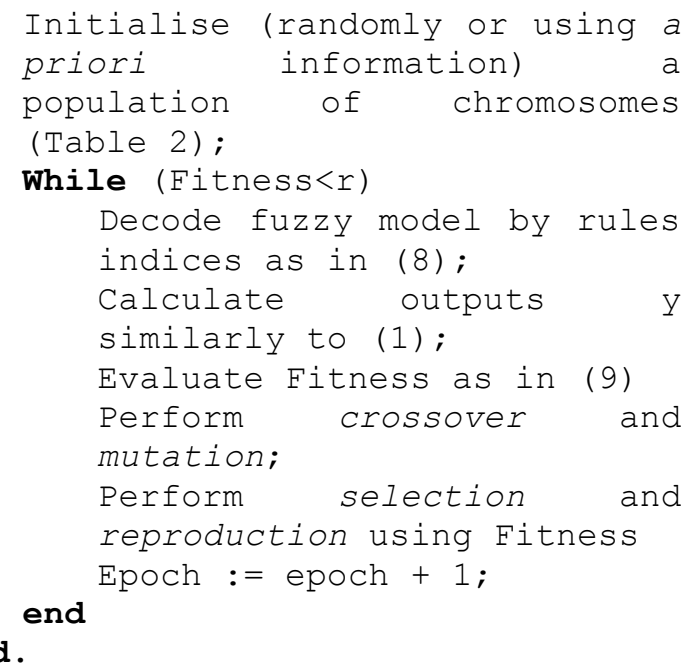

End.

where

$\mathrm{r}$ is a pre-defined desired correlation value;

Epoch denotes number of epochs.

The algorithm is initialised by a set of chromosomes (population), which is randomly seeded or defined on the base of a priori expert knowledge and previous experience (if such exists). Child chromosomes are produced using modified recombination, mutation and selection operation. They are performed separately for both parts (Table 2) because of the specific of the problem: a part of chromosomes represents indices of the rules and consists of integer values while the other part represents the parameters of fuzzy sets and consists of real values. Mutation over first part of the chromosomes, which contains the integer values, is performed with size of mutation step [11] equal to 1 such that to produce an integer number again. The selection is performed for the whole chromosomes because both parts contribute to the fitness value.

It has been demonstrated to the authors that when the number of rules is restricted, it is possible that not all the FLT are represented, and hence "holes" in the input/output space can be evident. While the application of overlapping Gaussian membership functions can minimise the effect on the model, gaps in the coverage of the model are still undesirable. To reduce the likelihood of holes being present a penalty function has been introduced that checks the population of solutions penalises these proportionally to the number of holes present. The penalty function is implemented in the fitness function by,

$$
f=\exp \left(-\sum_{\mathrm{i}=1}^{\mathrm{n}}\left(\mathrm{y}_{\mathrm{i}}-\hat{\mathrm{y}}_{\mathrm{i}}\right)^{2}-\Delta-\kappa\right),
$$

where $\kappa$ is the number of holes and $\Delta$ is given by,

$$
\Delta=\max \left\{\max _{i=1}^{\mathrm{n}}\left(\mathrm{y}_{\mathrm{i}}-\hat{\mathrm{y}}_{\mathrm{i}}\right)-\min _{\mathrm{i}=1}^{\mathrm{n}}\left(\mathrm{y}_{\mathrm{i}}-\hat{\mathrm{y}}_{\mathrm{i}}\right)\right\}
$$

In the example used in this paper the function ensured that the model gave complete coverage of the data, without the function, one or more holes were usually present in the optimal solution. In addition, if the number of rules required is too small to ensure complete coverage of the input/output space by the FLT, the penalty function will still allow feasible solutions to be derived, but will favour the solutions with the least number of holes.

\section{MODELLING A BOILER}

A practical problem of modelling of small boiler that could be used to supply medium temperature hot water to a heating system is considered.

A first principles based model of a nominally rated $300 \mathrm{~kW}$, natural gas fired boiler was used to generate training data that covered a typical range of operation. The boiler was assumed to operate at a constant water mass flow rate and a flow and return water temperature difference of $15 \mathrm{~K}$. Control of boiler operation is typically based on the return water temperature, thus the firing rate and return water temperature were excited to generate the input data. For use in HVAC system simulation, the model inputs were considered to vary between the maximum firing rate down to $10 \%$ of that rate, and for return water temperatures between $20^{\circ} \mathrm{C}$ and $100^{\circ} \mathrm{C}$. The resulting FRB model is therefore suitable when the boiler is firing, the water mass flow rate through the boiler is a constant $3.8 \mathrm{kgS}^{-1}$ and for normal operating conditions as well as "start-up" operation with the heating fluid at ambient temperatures.

From the data, two models were generated. The first predicted the gross efficiency of the boiler, taking the boiler load and return water temperature as inputs. The second modelled the flow temperature as the output as a function of the return water temperature and the control signal (percent of the maximum firing rate) to the boiler. The latter model allows the incorporation of the component into a subsystem performance simulation, while the former model can be used in conjunction with this to generate predictions of fuel consumption for energy analysis, such as annual energy cost predictions. Figure 1 demonstrates this hybrid approach the problem solution. "FP" refers to "First Principles" meaning here, a directly calculable algebraic relationship based on the physical relationship of the variables. 


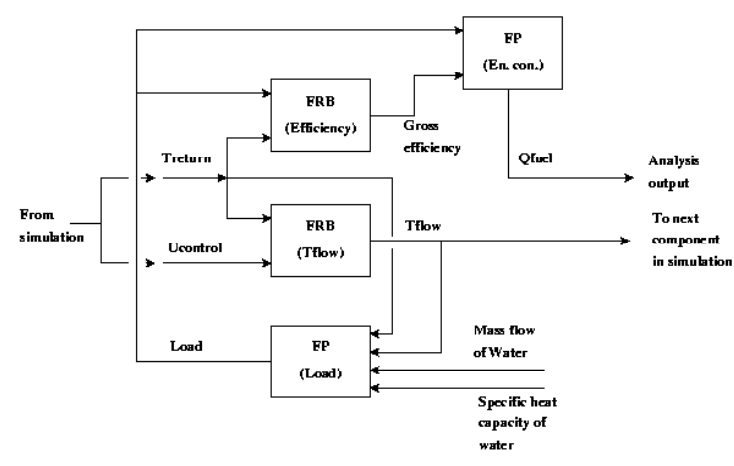

Fig 1. Hybrid Boiler Model Scheme.

In the scheme in Figure 1, the load model (FP: Load) is given by,

$$
q=\dot{m}_{w} C p_{w}\left(T_{\text {flow }}-T_{\text {retrum }}\right)
$$

and the fuel consumption (FP: En. Con.) model is given by,

$$
Q_{\text {fuel }}=\frac{q}{\eta_{\text {gross }}}
$$

where $q$ is the load across the boiler, $\dot{m}_{w}$ and $C p_{w}$ are the mass flow rate and specific heat capacity of water. $T_{\text {flow }}$ and $T_{\text {retrum }}$, are the flow and return water temperatures respectively. $Q_{\text {fuel }}$ and $\eta_{\text {gross }}$ are the instantaneous fuel consumption, in $\mathrm{kJS}^{-1}$ and the gross efficiency of the boiler.

In each case 7 FLTs and 20 rules where used to characterise the process. The training and validation results for both the efficiency and the flow temperature models are depicted in Figures 2 and 3.
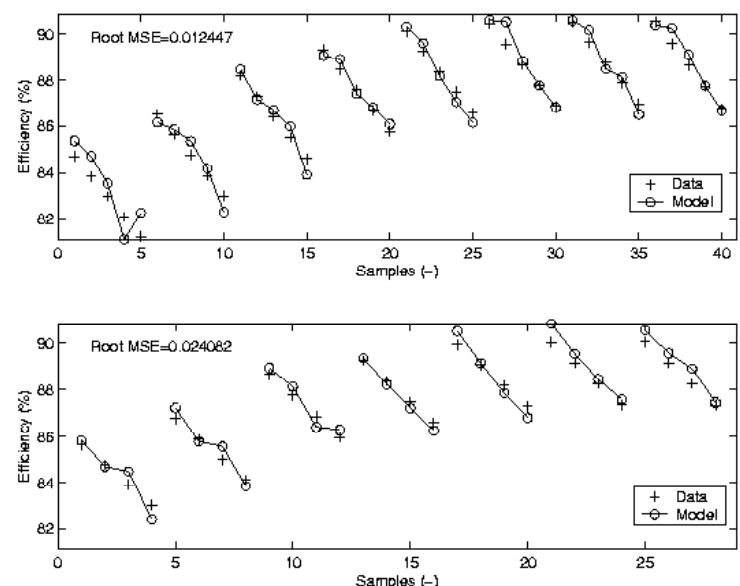

Fig 2. Sample-wise plots of the Efficiency model predictions for training and validation data.
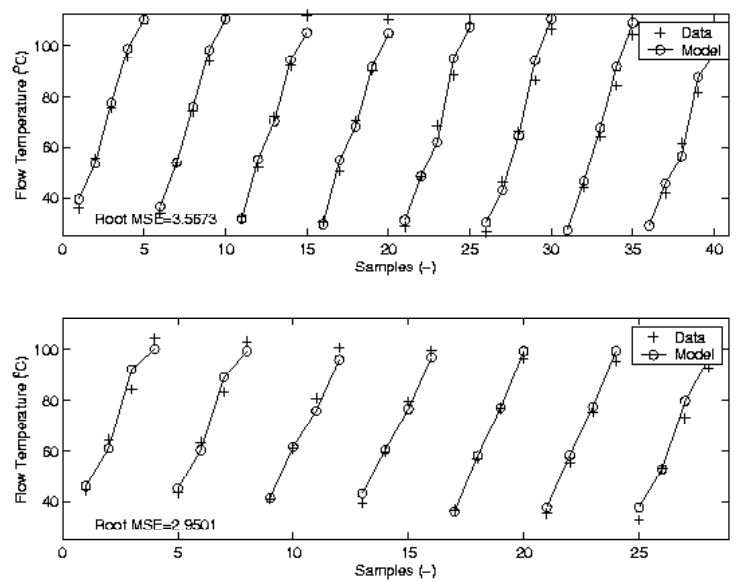

Fig 3. Sample-wise plots of the Temperature model predictions for training and validation data.

The term "Root MSE" noted on each plot refers to the root-mean-square-error between the data and model predictions. The product-momentcorrelation-coefficients between the measured and predicted values for each plot are shown in Table III.

TABLE III

The Product Moment Correlation COEFFicients for THE BOILER MODELS

\begin{tabular}{|c|c|c|}
\hline Model & Training & Validation \\
\hline Gross Efficiency & 0.982 & 0.986 \\
\hline Flow Temperature & 0.990 & 0.988 \\
\hline
\end{tabular}

Both models have quite small errors and very high correlation's, although some improvement is possible be extending the time allowed to yield the optimum solution. The efficiency model was generated at 2000 generations and the flow temperature model at $\sim 1000$. The first principle model used to generate the data has a smooth input/output surface and both FRB models produced quite smooth function surfaces without the application of a smoothing function. This would undoubtedly improve the model, especially in respect of the amount of data required to characterise the input/output space.

\section{CONCLUSION}

A methodology for the evolutionary construction of fuzzy rule-based (EFRB) models is proposed in the paper. The resulting models are transparent and existing expert knowledge could easily be incorporated into the model (both at initialisation stages and during its generation). An additional advantage of the model is represented by the economy in computational effort in generating the model output. A new encoding mechanism is used that allows the fuzzy model rule base structure and parameters to be estimated from training data 
without establishing the complete rule list. It uses rule indices and therefore significantly reduces the computational load. The rules are extracted from the data without using a priori information about the inherent model structure. It makes EFRB models as flexible as other types of 'black-box' models (neural networks, polynomial models etc.) and in the same time significantly more transparent, especially when only small subset of all possible rules is considered. Practical building services engineering problem is considered in order to illustrate the applicability of the approach. The EFRB models have potential applications in simulation, control and fault detection and diagnosis.

\section{ACKNOWLEDGEMENT}

The authors would like to acknowledge the support by the EPSRC by the grant GR/M97299.

\section{REFERENCES}

1 Angelov P., Evolving Fuzzy Rule-based Models, 8th IFSA World Congress, Taipei, Taiwan, August 1999, pp.19-23

2 Diaz G., M. Sen, K. T. Yang, R. L. McClain, Simulation of Heat Exchanger Performance by Artificial Neural Networks, HVAC\&R Research, v.5 (3), 195-208 (1999).

3 Bastian A., A Genetic Algorithm for Tuning Membership Functions, 4th Euro Congress on Fuzzy and Intelligent Technologies EUFIT'96, Aachen, Germany, 1, 494-498 (1996).

4 Carse B., T.C. Fogarty, A. Munro, Evolving fuzzy rule-based controllers using GA, Fuzzy Sets and Systems, 80, 273-294 (1996).

5 Chiang C. K., H.-Y. Chung, J.J. Lin, A SelfLearning Fuzzy Logic Controller using Genetic Algorithms with Reinforcements, IEEE Transactions on Fuzzy Systems, 5, 460467 (1996).

6 Yager R., D. Filev, Essentials of Fuzzy Modeling and Control, John Willey and Sons, NY (1994).

7 Goldberg D. E., Genetic Algorithms in Search, Optimization and machine Learning, AddisonWesley, Reading, MA (1989).

8 Hanby V. I., J.A. Wright, HVAC Modelling Studies, Building Services Engineering Research and Technology, 10, 35-39 (1989).

9 Lim M. H., S.Rahardja, B.H.Gwee, A GA Paradigm for Learning Fuzzy Rules, Fuzzy Sets and Systems, 82, 177-186 (1996).

10 Michalewicz Z., Genetic Algorithms + Data Structures $=$ Evolution Programs, Springer Verlag, Berlin (1996).

11 Muhlenbein H., D. Schlierkamp-Voosen, Predictive Models for the Breeder GA, Evolutionary Computation, 1, 25-49 (1993).
12 Nelles O., FUREGA - Fuzzy Rule Extraction by GA, Proc. of the 4th European Congress on Fuzzy and Intelligent Technologies EUFIT'96, Aachen, Germany, 1, 489-493 (1996).

13 Nozaki K., T. Morisawa, H. Ishibuchi, Adjusting Membership Functions in Fuzzy Rule-based Classification Systems, Proc. of the $3 d$ European Congress on Fuzzy and Intelligent Technologies, EUFIT'95, Aachen, Germany, 1, 615-619 (1995). 